BRAGA, MB; MAROUELLI, WA; RESENDE, GM; MOURA, MSB; COSTA, ND; CALGARO, M; CORREIA, JS. 2017. Coberturas do solo e uso de manta agrotêxtil (TNT) no cultivo do meloeiro. Horticultura Brasileira 35: 147-153. DOI - http://dx.doi.org/10.1590/S0102-053620170123

\title{
Coberturas do solo e uso de manta agrotêxtil (TNT) no cultivo do meloeiro
}

\author{
Marcos B Braga ${ }^{1}$; Waldir A Marouelli ${ }^{1}$; Geraldo M Resende²; Magna SB Moura²; Nivaldo D Costa ${ }^{2}$; \\ Marcelo Calgaro' ${ }^{2}$ Joselina S Correia ${ }^{3}$ \\ 'Embrapa Hortaliças, Brasília-DF, Brasil; marcos.braga@embrapa.br; waldir.marouelli@embrapa.br; ²Embrapa Semiárido, Petrolina- \\ -PE, Brasil; geraldo.milanez@embrapa.br; magna.moura@embrapa.br; nivaldo.duarte@embrapa.br; marcelo.calgaro@embrapa.br; \\ ${ }^{3}$ Universidade Estadual Paulista (UNESP), Botucatu-SP, Brasil; linajua@hotmail.com
}

\begin{abstract}
RESUMO
O uso de cobertura do solo na produção de melão pode ser uma alternativa na região do Submédio do Vale do São Francisco, onde o grande desafio é produzir em quantidade e qualidade na entressafra. O objetivo foi determinar o efeito de coberturas de solo e manta agrotêxtil (TNT) na produtividade e qualidade dos frutos do meloeiro na entressafra de cultivo (plantio no primeiro semestre) no Submédio do Vale do Rio São Francisco, Petrolina-PE. O experimento foi conduzido nos anos de 2009 e 2010, durante junho a agosto. O delineamento foi em blocos casualizados com três repetições, em arranjo fatorial 6x2. Os tratamentos compreenderam cinco tipos de coberturas do solo (plástico preto, plástico dupla face preto/prata, casca de coco, palha de capim buffel, bagaço de cana) e a testemunha em solo descoberto, com a presença ou ausência de TNT. O número de frutos por planta variou entre 1,11 e 1,70, sem diferenças significativas entre tratamentos, à exceção do tratamento com casca de coco que apresentou pior desempenho em 2010. A maior e menor produção de frutos por planta foi obtida pela cobertura com polietileno de dupla face e solo descoberto (2,91 e 1,91 kg/planta, respectivamente). As coberturas do solo com polietileno de dupla face, palha de capim buffel e bagaço de cana apresentaram produtividade comercial superior ou similar aos demais tratamentos variando entre 35,60 e 58,20 t/ha. De forma geral, as características físicas e de qualidade do fruto pouco foram influenciadas pelas coberturas do solo e TNT. Considerando os dois ciclos de cultivo, os tratamentos polietileno de dupla face, bagaço de cana, palha de capim buffel, polietileno preto e casca de coco sobressaíram com melhor índice de produtividade da água, variando entre 113,8 e 133,9 L de água/kg massa fresca de fruto e o solo descoberto apresentou valor pior $(164,1 \mathrm{~L} / \mathrm{kg})$.
\end{abstract}

Palavras-chave: Cucumis melo, mulching, cobertura da planta, qualidade de frutos, produtividade.

\begin{abstract}
Soil covers on the melon cultivation under nonwoven blanket

The use of ground cover in melon production could be an alternative in the region of the São Francisco Valley, where the challenge is to produce in quantity and quality in the off season. This study aimed to evaluate the influence of five soil cover types, with and without nonwoven blanket (TNT) cover on productivity and quality of melon fruits in non-traditional growing season in the São Francisco River region, Petrolina, Pernambuco State, Brazil. The experiment was carried out in 2009 and 2010, during June to August. Experimental design was randomized blocks with three replications in a factorial arrangement $6 \times 2$. Treatments included five types of soil cover (black polyethylene and black-silvery double face polyethylene film, coconut shell, buffelgrass straw and sugarcane bagasse), uncovered soil treatment, and the plant covers previously mentioned with or without TNT. The number of fruits per plant ranged from 1.11 to 1.70 fruits without significant differences between treatments, except for the treatment with coconut shell which showed a worse performance in 2010. The highest and lowest production of fruits per plant were obtained using black-silvery double face polyethylene film and uncovered soil (2.91 and $1.91 \mathrm{~kg} / \mathrm{plant}$, respectively). The soil covers black-silvery double face polyethylene film, buffelgrass straw and sugarcane bagasse showed higher marketable yield or similar to the other treatments ranging between 35.60 and $58.20 \mathrm{t} /$ ha. In general, the physical characteristics and fruit quality were little influenced by soil cover and TNT. In terms of water productivity index (iPA), considering the two crop cycles, black-silvery double face polyethylene film, sugarcane bagasse, buffelgrass straw, black polyethylene and coconut shell showed a better iPA, varying between 113.8 and $133.9 \mathrm{~L} / \mathrm{kg}$ of fresh fruit, while uncovered soil showed a worse value $(164.1 \mathrm{~L} / \mathrm{kg})$.
\end{abstract}

Keywords: Cucumis melo, mulching, plant cover, fruit quality, yield.

(Recebido para publicação 3 de dezembro de 2015; aceito em 25 de julho de 2016) (Received on December 3, 2015; accepted on July 25, 2016)

$\mathrm{O}$ melão é uma das hortaliças de maior importância no comércio exterior do Brasil, tendo sido exportado, principalmente para a Europa, $177,8 \mathrm{mil}$ toneladas de frutos no ano de 2010 , gerando divisas da ordem de 122 milhões de dólares. Da produção nacional, que em 2010 foi de 478,4 mil toneladas, a região Nordeste foi responsável por aproximadamente $95 \%$, sendo a Chapada do Apodi-RN e o Baixo Jaguaribe-CE, as principais regiões produtoras
(Agrianual, 2013).

O uso de novas tecnologias, associado ao aprimoramento global de todo o sistema de produção da cultura, são fundamentais para o sucesso no cultivo de melão no semiárido brasileiro. 
Medeiros et al. (2006) relatam que o incremento na produtividade e a melhoria na qualidade do melão produzido são devidos, entre outras variáveis, ao desenvolvimento de tecnologias adaptadas para cada região produtora, incluindo, mais recentemente, o uso de agrotêxteis, também conhecido como tecido não tecido (TNT) para cobertura das plantas no início do ciclo de cultivo com foco de proteção contra insetos e estímulo do crescimento inicial. A manta agrotêxtil (TNT) vem sendo usada nas regiões produtoras de melão no Nordeste brasileiro com intuito de amenizar os ataques de insetos, principalmente, o da mosca branca, que é indicado como o principal transmissor de viroses para a cultura do meloeiro (Santos, 2012). Dias et al. (2006), confirmado por Braga et al. (2010), para as condições edafoclimáticas dos estudos, não encontraram efeitos significativos do uso do TNT na produtividade do meloeiro amarelo.

Outra tecnologia de produção de plantas é a cobertura do solo que possibilita ganhos em produtividade e redução de impactos ambientais. Segundo Câmara et al. (2007) e Braga et al. (2010), o uso de cobertura de solo possibilita reduzir a oscilação da temperatura no solo, evaporação de água do solo e a perda de adubos e corretivos por lixiviação, minimizando a compactação, a erosão, o contato direto dos frutos com o solo, os danos dos frutos e melhorando sua aparência visual.

A cobertura parcial do solo com material orgânico ou com filmes de polímeros plásticos vem sendo utilizada em todo o mundo, principalmente na produção de hortaliças, com ganhos notórios tanto em produtividade, quanto na redução dos custos de produção (Brandenberger \& Wiedenfeld, 1997). Orozco et al. (1995) verificaram aumento expressivo na produtividade de melão cultivado em solo parcialmente coberto com plástico de polietileno branco (31 t/ha) em relação ao solo descoberto (7 t/ha). Battikhi \& Ghawi (1987) também obtiveram ganhos expressivos na produtividade de melão quando o solo foi parcialmente coberto com mantas de plástico preto (29 t/ha) e de plástico branco (14 t/ha), comparativamente ao cultivo em solo sem cobertura (6 t/ ha). Em estudo realizado no semiárido brasileiro com melão amarelo, Braga et al. (2010) verificaram que a produtividade e a eficiência do uso de água com a cobertura do solo, incluindo diferentes tipos de materiais orgânicos e de filmes de polímeros plásticos, foram significativamente maiores que em solo descoberto. Dentre os tipos de cobertura, palha de capim buffel possibilitou maior produtividade (73 t/ha), 27\% acima daquela verificada em solo sem cobertura.

Em regiões áridas e semiáridas, devido à escassez hídrica, o uso racional e eficiente da água é essencial para a maior sustentabilidade da agricultura irrigada. Assim, um dos principais benefícios do uso de cobertura de solo em cultivos irrigados é a redução das perdas de água por evaporação e maior conservação da umidade do solo (Miranda et al., 2003; Braga et al., 2010). Moura et al. (2009) destacam que, além de reduzir a evaporação de água, o uso de cobertura do solo reduz a variação da amplitude térmica do solo. Adicionalmente ao ganho de produtividade e redução no uso de água, Amariz et al. (2009) relatam que o uso de cobertura do solo pode favorecer a conservação de frutos de melão, aspecto fundamental durante $\mathrm{o}$ transporte $\mathrm{e} \mathrm{o}$ armazenamento dos mesmos.

Um dos grandes desafios dos produtores de melão na região do Submédio do Vale do São Francisco é produzir melões em quantidade e qualidade em plantio no primeiro semestre do ano (entressafra), uma vez que, neste período há a ocorrência de intempéries climáticas, como a ocorrência de frio e chuvas, o que afeta o desenvolvimento da cultura, além de aumentar a incidência de doenças, que dificultam a produção econômica da cultura.

Esse trabalho teve como objetivo avaliar a produtividade e qualidade do melão cultivado sobre diferentes tipos de cobertura do solo, com uso temporário de manta agrotêxtil em cultivo de entressafra, no Submédio do Vale do São Francisco.

\section{MATERIAL E MÉTODOS}

Conduziu-se o experimento no
Campo Experimental de Bebedouro (09 $09^{\prime} \mathrm{S}, 40^{\circ} 22^{\prime} \mathrm{O}$, altitude $366 \mathrm{~m}$ ), Petrolina-PE, pertencente à Embrapa Semiárido, nos anos 2009 e 2010. O clima da região, segundo a classificação de Köppen, é do tipo BSwh, ou seja, semiárido com temperaturas médias anuais elevadas $\left(26,1^{\circ} \mathrm{C}\right)$ e precipitação média de $515 \mathrm{~mm}$. Em 2009, o período do experimento foi de $18 / 06$ a 24/08 com temperaturas médias máximas e mínimas de 31,8 e $19,3^{\circ} \mathrm{C}$, respectivamente. A precipitação acumulada nesse período foi de 19,1 mm. Em 2010 realizou-se o transplantio das mudas em 08/06 e a colheita em 17/08; nesse período as temperaturas médias máximas e mínimas foram de 31,1 e $19^{\circ} \mathrm{C}$, respectivamente e a precipitação acumulada foi de 26,2 mm. Esses dados foram coletados de uma estação agrometeorológica localizadas a $600 \mathrm{~m}$ da área do ensaio experimental.

O solo da área experimental é classificado como Latossolo Vermelho Amarelo de textura arenosa (86,05\% areia total; $7,85 \%$ silte e $6,1 \%$ argila). Em 2009, a análise química do solo revelou: matéria orgânica $=10,38 \mathrm{~g} / \mathrm{kg}$; $\mathrm{pH}$ (água) $=6,33 ; \mathrm{P}_{\text {(Mehlich) }}=24,62 \mathrm{mg} /$ $\mathrm{dm}^{3} ; \mathrm{K}, \mathrm{Ca}, \mathrm{Mg}=0,31 ; 1,60$ e $1 \mathrm{cmolc} /$ $\mathrm{dm}^{3}$ respectivamente; condutividade elétrica (CE) do extrato de saturação de $0,62 \mathrm{dS} / \mathrm{m} ; \mathrm{V} \%=98,32 \%$ e $\mathrm{CTC}=$ $2,97 \mathrm{cmolc} / \mathrm{dm}^{3}$. Os valores de $\mathrm{Cu}, \mathrm{Fe}$, $\mathrm{Mn}$ e $\mathrm{Zn}$ foram respectivamente 0,64 ; 23,$93 ; 1,04$; e $25,87 \mathrm{mg} / \mathrm{dm}^{3}$. Em 2010 os resultados da análise química do solo revelaram: matéria orgânica $=8,69 \mathrm{~g}$ / $\mathrm{kg} ; \mathrm{pH}_{\text {(água) }}=6,1 ; \mathrm{P}_{\text {(Menlich) }}=37,44 \mathrm{mg} /$ $\mathrm{dm}^{3} ; \mathrm{K}, \mathrm{Ca}, \mathrm{Mg}=0,32 ; 2,3$ e $1,2 \mathrm{cmolc} /$ $\mathrm{dm}^{3}$, respectivamente; $C E$ do extrato de saturação $=0,30 \mathrm{dS} / \mathrm{m} ; \mathrm{V} \%=97,88 \%$ e $\mathrm{CTC}=3,92 \mathrm{cmolc} / \mathrm{dm}^{3}$. Os valores de $\mathrm{Cu}, \mathrm{Fe}, \mathrm{Mn}$ e $\mathrm{Zn}$ foram respectivamente, 1,$10 ; 50 ; 1,92$ e $27,73 \mathrm{mg} / \mathrm{dm}^{3}$.

Utilizou-se o delineamento de blocos ao acaso, em esquema fatorial $6 \times 2$, compreendendo seis tratamentos (coberturas de solo com casca de coco seco (CC), palha de capim buffel (PCB) e bagaço de cana (BC) com $2 \mathrm{~cm}$ de espessura, plástico preto ( $\mathrm{PP})$, plástico duplo face preto/prata (PDF) com 30 micra de espessura e solo descoberto sem qualquer tipo de cobertura morta (SD), usado como controle e dois usos 
de manta agrotêxtil (TNT) (com e sem), com seis repetições.

Cultivaram-se os híbridos SF 10/00 F1 e o Tropical F1 (Agristar/Topseed), ambos amarelos, nos anos de 2009 e 2010, respectivamente. Produziram-se as mudas em bandejas de 128 células e as transplantaram aos 14 dias após a semeadura em junho de 2009 e 2010 no espaçamento de 2,0 m entre linhas e $0,25 \mathrm{~m}$ entre plantas. Assim, a cultura foi conduzida durante o período da entressafra do melão na região, ou seja, na estação com maior ocorrência de temperaturas mais amenas (frio). A unidade experimental constou de três linhas de $6 \mathrm{~m}$ de comprimento espaçadas de $2 \mathrm{~m}$, perfazendo um total $36 \mathrm{~m}^{2}$. Para a análise de produção foram avaliados os frutos de 4,0 $\mathrm{m}$ da fileira central de plantas (área útil de $8 \mathrm{~m}^{2}$ ).

Realizou-se a adubação mineral com base na análise de solo seguindo recomendações de Crisóstomo et al. (2002). Foram aplicados $120 \mathrm{~kg} / \mathrm{hade}$ nitrogênio (N), $240 \mathrm{~kg} /$ ha de fósforo $\left(\mathrm{P}_{2} \mathrm{O}_{5}\right)$ e 180 $\mathrm{kg}$ /ha de potássio $\left(\mathrm{K}_{2} \mathrm{O}\right)$ em 2009 , e 120 $\mathrm{kg} / \mathrm{ha}$ de N, $180 \mathrm{~kg} / \mathrm{ha} \mathrm{de} \mathrm{P}_{2} \mathrm{O}_{5}$ e $180 \mathrm{~kg} /$ ha de $\mathrm{K}_{2} \mathrm{O}$ em 2010. A adubação fosfatada (superfosfato simples) foi realizada de forma convencional por ocasião do transplante, enquanto a adubação nitrogenada (ureia) e potássica (sulfato de potássio) foi realizada três vezes por semana via fertirrigação.

A colocação dos diferentes filmes plásticos e materiais orgânicos sobre o solo foi feita após a instalação do sistema de irrigação e antes do transplante. Cobriu-se uma faixa de solo de $1,0 \mathrm{~m}$ ao longo da fileira de plantas, o que representou uma cobertura de 50\% da área de cultivo. Os filmes plásticos usados tinham espessura de 30 micra e a espessura média da camada de material orgânico sobre o solo foi de $2 \mathrm{~cm}$.

Imediatamente após o transplante realizou-se pulverização preventiva com fungicida para em seguida instalar o túnel de TNT (cor branca, com gramatura de $15 \mathrm{~g} / \mathrm{m}^{2}$ ) sobre as mudas das parcelas dos tratamentos que receberam a cobertura com TNT durante os primeiros 20 dias de cultivo do meloeiro. Instalou-se a cobertura sobre arcos de ferro com $50 \mathrm{~cm}$ de altura, $70 \mathrm{~cm}$ de largura na base e distanciados a cada $7 \mathrm{~m}$ (Pires et al., 2013).

Utilizou-se sistema de irrigação por gotejamento, com gotejadores com vazão de $2,65 \mathrm{~L} / \mathrm{h}$, espaçados de 50 $\mathrm{cm}$. Realizaram-se irrigações diárias e igualitárias por tratamento, com lâmina de água aplicada estimada com base na evaporação de tanque Classe "A" e coeficientes de cultura propostos por Sousa et al. (2000).

Registrou-se a temperatura do solo por meio da instalação de termopares tipo $\mathrm{T}$ (copper-constantan), a 5 e $10 \mathrm{~cm}$ de profundidade, entre duas plantas, no centro de duas parcelas experimentais de cada tratamento. Os sensores foram conectados a um sistema de aquisição de dados, modelo CR800 (Campbell Scientific, Logan, USA), programado para armazenar temperaturas médias a cada hora, durante todo o ciclo de cultivo do meloeiro.

Os dados de temperatura do ar, umidade relativa do ar e precipitação no período de condução dos experimentos foram obtidos na estação agrometeorológica da Embrapa Semiárido, instalada no Campo Experimental de Bebedouro, localizada a 500 metros da área experimental.

Iniciaram-se as colheitas quando os frutos apresentavam coloração da casca amarelada e teor de sólidos solúveis totais acima de $9^{\circ}$ Brix. Em 2009 realizaram-se duas colheitas, aos 71 dias e 85 dias após o plantio, enquanto em 2010 realizou-se somente uma colheita 72 dias após o plantio.

Após a colheita dos frutos avaliou-se o número de frutos por planta, massa fresca de fruto por planta, diâmetros externos equatorial (DEquat) e longitudinal (DLong), diâmetros das cavidades equatorial (DCEq) e longitudinal (DCLong), espessuras de polpa (EPolp) e de casca (Ecasca), sólidos solúveis totais (SST), $\mathrm{pH}$, acidez total titulável e índice de produtividade da água (iPA). As análises das variáveis físicas e de qualidade foram realizadas no Laboratório de Pós-colheita da Embrapa Semiárido, onde foram sorteados cinco frutos ao acaso, em cada parcela. Para analises dos frutos usou-se a metodologia descrita pelo Instituto Adolfo Lutz (1985). O iPA foi determinado conforme Jensen (2007), ou seja, pela relação entre o volume total de água aplicado via irrigação e a produção comercial de frutos de melão por unidade de área.

Os dados foram submetidos à análise de variância (teste $F, p \leq 0,05$ ) e as médias das variáveis comparadas pelo teste de Tukey a $5 \%$ de probabilidade, usando o programa SISVAR 5.1 (Ferreira, 2008).

\section{RESULTADOS E DISCUSSÃO}

\section{Plantio no primeiro semestre de 2009}

Não houve interação significativa entre tratamentos de cobertura do solo e uso da manta agrotêxtil nas características estudadas, assim como o uso da manta não influenciou o número de frutos e produção por planta e produtividade do melão.

Para número de frutos por planta, não houve diferenças significativas entre tratamentos, sendo verificados valores entre 1,38 e 1,70 frutos (Tabela 1). A maior produção de frutos por planta foi obtida pelo tratamento com cobertura de polietileno de dupla face (PDF) $(2,91$ $\mathrm{kg} / \mathrm{planta})$, que não diferiu dos tratamentos com bagaço de cana (BC) e palha de capim buffel (PCB), respectivamente de 2,34 e 2,16 kg/planta (Tabela 1).

Resultados similares foram observados para produtividade comercial do melão, com destaque para a cobertura com PDF que obteve o melhor resultado com 58,2 t/ha, no entanto, sem diferenciar dos tratamentos $\mathrm{BC}$ e $\mathrm{PCB}$ (Tabela 1). Resultados semelhantes foram encontrados por Brandenberger \& Wiedenfeld (1997), Orozco et al. (1995) e Battikhi \& Ghawi (1987) que comprovaram a eficiência do mulching na produção do meloeiro em relação ao controle. Braga et al. (2010) verificaram diferenças significativas para todos os tratamentos em relação à produtividade, fato este justificado devido à realização do cultivo no segundo semestre de 2008 , período de safra do melão e de condição agroclimática favorável à produção (maiores temperaturas). Mesmo assim, as produtividades obtidas estão acima das principais regiões produtoras do país, onde se consegue produzir entre 24,8 a 34,4 t/ha (Crisóstomo et al., 2002) e também acima da média brasileira 
Tabela 1. Número de frutos, produção de frutos por planta e produtividade comercial de melão em diferentes tipos de cobertura do solo (number of fruits, yield per plant and commercial yield of melon under different types of soil covers). Petrolina, Embrapa Semiárido, $2009-2010$.

\begin{tabular}{lccc}
\hline \multirow{2}{*}{ Tratamentos } & $\mathbf{N}^{\mathbf{0}}$ de frutos por planta & Produção de frutos (kg/planta) & Produtividade (t/ha) \\
\cline { 2 - 4 } & \multicolumn{3}{c}{$\mathbf{2 0 0 9}$} \\
\hline Palha de capim buffel & $1,58 \mathrm{a}$ & $2,16 \mathrm{ab}$ & $43,20 \mathrm{ab}$ \\
Casca de coco & $1,50 \mathrm{a}$ & $1,93 \mathrm{~b}$ & $38,60 \mathrm{~b}$ \\
Polietileno preto & $1,41 \mathrm{a}$ & $2,11 \mathrm{~b}$ & $42,20 \mathrm{~b}$ \\
Bagaço de cana & $1,53 \mathrm{a}$ & $2,35 \mathrm{ab}$ & $47,00 \mathrm{ab}$ \\
Polietileno dupla face & $1,70 \mathrm{a}$ & $2,91 \mathrm{a}$ & $58,20 \mathrm{a}$ \\
Solo descoberto & $1,38 \mathrm{a}$ & $1,92 \mathrm{~b}$ & $38,40 \mathrm{~b}$ \\
\hline CV (\%) & 13,03 & 19,69 & 19,70 \\
\hline & & $\mathbf{2 0 1 0}$ & $38,20 \mathrm{a}$ \\
\hline Palha de capim buffel & $1,37 \mathrm{a}$ & $1,91 \mathrm{a}$ & $37,60 \mathrm{a}$ \\
Casca de coco & $1,19 \mathrm{a}$ & $1,88 \mathrm{a}$ & $36,80 \mathrm{ab}$ \\
Polietileno preto & $1,11 \mathrm{~b}$ & $1,84 \mathrm{ab}$ & $35,80 \mathrm{ab}$ \\
Bagaço de cana & $1,26 \mathrm{a}$ & $1,79 \mathrm{ab}$ & $35,60 \mathrm{ab}$ \\
Polietileno dupla face & $1,13 \mathrm{a}$ & $1,78 \mathrm{ab}$ & $25,60 \mathrm{~b}$ \\
Solo descoberto & $1,27 \mathrm{a}$ & $1,28 \mathrm{~b}$ & 18,96 \\
\hline CV (\%) & 11,32 & 18,90 & $\mathrm{~b}$ \\
\hline
\end{tabular}

*Médias seguidas da mesma letra na coluna não diferem entre si pelo teste de Tukey, ao nível de 5\% de probabilidade (means followed by the same letter in the column do not differ significantly, Tukey 0.05 ).

Tabela 2. Diâmetro equatorial (DEquat); diâmetro longitudinal (DLong); diâmetro equatorial da cavidade (DCEq); diâmetro longitudinal da cavidade (DCLong); espessura de polpa (EPolp); espessura de casca (Ecasca) em função de diferentes tipos de coberturas de solo e uso de manta agrotêxtil (TNT) \{equatorial diameter (DEquat); longitudinal diameter (DLong); equatorial diameter of the cavity (DCEq); longitudinal diameter of the cavity (DCLong); flesh thickness (EPolp); shell thickness (Ecasca) depending on different soil cover types and use of nonwoven blanket (TNT)\}. Petrolina, Embrapa Semiárido, 2010.

\begin{tabular}{lcccccc}
\hline Tratamentos & $\begin{array}{c}\text { DEquat } \\
(\mathbf{c m})\end{array}$ & $\begin{array}{c}\text { DLong } \\
(\mathbf{c m})\end{array}$ & $\begin{array}{c}\text { DCEq } \\
(\mathbf{c m})\end{array}$ & $\begin{array}{c}\text { DCLong } \\
(\mathbf{c m})\end{array}$ & $\begin{array}{c}\text { EPolp } \\
(\mathbf{c m})\end{array}$ & $\begin{array}{c}\text { ECasca } \\
(\mathbf{c m})\end{array}$ \\
\hline Palha capim buffel & $13,68 \mathrm{a}$ & $17,20 \mathrm{ab}$ & $5,88 \mathrm{a}$ & $12,21 \mathrm{a}$ & $3,69 \mathrm{a}$ & $0,57 \mathrm{a}$ \\
Casca de coco & $13,28 \mathrm{a}$ & $16,99 \mathrm{ab}$ & $6,05 \mathrm{a}$ & $11,92 \mathrm{a}$ & $3,70 \mathrm{a}$ & $0,55 \mathrm{a}$ \\
Polietileno preto & $13,47 \mathrm{a}$ & $17,19 \mathrm{ab}$ & $5,55 \mathrm{a}$ & $12,15 \mathrm{a}$ & $3,87 \mathrm{a}$ & $0,54 \mathrm{a}$ \\
Bagaço de cana & $13,33 \mathrm{a}$ & $17,41 \mathrm{a}$ & $6,06 \mathrm{a}$ & $11,99 \mathrm{a}$ & $3,59 \mathrm{a}$ & $0,42 \mathrm{ab}$ \\
Polietil. dupla face & $13,38 \mathrm{a}$ & $17,07 \mathrm{ab}$ & $5,76 \mathrm{a}$ & $12,00 \mathrm{a}$ & $3,76 \mathrm{a}$ & $0,48 \mathrm{ab}$ \\
Solo descoberto & $13,17 \mathrm{a}$ & $16,05 \mathrm{~b}$ & $6,12 \mathrm{a}$ & $10,71 \mathrm{a}$ & $3,35 \mathrm{a}$ & $0,36 \mathrm{~b}$ \\
Sem manta TNT & $33,47 \mathrm{a}$ & $16,85 \mathrm{a}$ & $5,69 \mathrm{~b}$ & $11,54 \mathrm{~b}$ & $3,82 \mathrm{a}$ & $0,45 \mathrm{~b}$ \\
Com manta TNT & $13,31 \mathrm{a}$ & $17,18 \mathrm{a}$ & $6,11 \mathrm{a}$ & $12,21 \mathrm{a}$ & $3,51 \mathrm{~b}$ & $0,53 \mathrm{a}$ \\
\hline CV $(\%)$ & 4,31 & 4,36 & 7,36 & 7,12 & 9,85 & 18,17 \\
\hline
\end{tabular}

*Médias seguidas da mesma letra na coluna não diferem entre si pelo teste de Tukey, ao nível de $5 \%$ de probabilidade (means followed by the same letter in the column do not differ significantly, Tukey 0.05 ).

de 23 t/ha (Agrianual, 2013). O uso da manta TNT não influenciou significativamente o número de frutos por planta e a produtividade do meloeiro; resultado semelhante foi encontrado por Dias et al. (2006).

Não foram observadas diferenças significativas entre tratamentos para diâmetro equatorial, longitudinal, da cavidade equatorial e espessura de casca, observando-se apenas para a espessura de polpa na qual o tratamento PDF sobressaiu-se com $3,43 \mathrm{~cm}$, porém sem diferir estatisticamente do tratamento de cobertura com plástico preto (PP). Ressalta-se que houve diferenças estatísticas para uso da manta TNT em cobertura, para os parâmetros diâmetro longitudinal $(17,40 \mathrm{~cm})$, da cavidade longitudinal $(11,54 \mathrm{~cm})$ e espessura de casca (4 mm) (dados não apresentados).

Com relação aos parâmetros de qualidade de frutos não foram encontradas diferenças significativas entre tratamentos para $\mathrm{pH}$ (média de 5,47) e acidez total titulável (média de $0,15 \%$ volume de ácido cítrico). No entanto, com uso da manta de TNT, o $\mathrm{pH}$ foi mais elevado, tendo apresentado valor médio de 5,5. Os sólidos solúveis totais (SST) 
Tabela 3. Índice de produtividade da água (iPA) pela cultura do melão em função de tipos de cobertura do solo \{water productivity index (iPA) by the melon crop depending on soil cover types $\}$. Petrolina, Embrapa Semiárido, 2009/2010.

\begin{tabular}{lcc}
\hline \multirow{2}{*}{ Tratamentos } & \multicolumn{2}{c}{ iPA (L/kg) } \\
\cline { 2 - 3 } & Ano 2009 & Ano 2010 \\
\hline Polietileno de dupla face & 46,42 & 67,33 \\
Bagaço de cana & 57,38 & 66,96 \\
Palha de capim buffel & 62,33 & 62,59 \\
Polietileno preto & 64,04 & 65,32 \\
Casca de coco & 70,00 & 63,92 \\
Solo descoberto & 70,41 & 93,64 \\
\hline
\end{tabular}

foram influenciados pelos tratamentos, com maiores valores para os tratamentos PDF $\left(11,8^{\circ}\right.$ Brix $), \operatorname{SD}\left(11,7^{\circ}\right.$ Brix $)$ e BC (10,7 $7^{\circ}$ rix), que não diferiram entre si. Para SST não foi encontrado efeito significativo do uso da manta TNT (média de $10,9^{\circ}$ Brix). Ressalta-se que os valores de SST para todos os tratamentos foram superiores a $9,0^{\circ} \mathrm{Brix}$, indicando que os melões produzidos em 2009 superaram os padrões de qualidade tanto no mercado interno quanto externo.

\section{0}

Plantio no primeiro semestre de

Não houve interação significativa entre tratamentos de cobertura do solo e uso da manta agrotêxtil nas características estudadas. No entanto, verificou-se diferença significativa entre os tratamentos de cobertura do solo para número de frutos por planta, com maior valor para PCB com 1,37 frutos, sem maiores produtividades, fato explicado devido a realização do cultivo ter sido no segundo semestre do ano (temperaturas mais elevadas), além da realização de três colheitas. Todavia, as produtividades obtidas no presente experimento, estão acima das médias das principais regiões produtoras do país.

Não houve efeito significativo do TNT em cobertura da cultura por 20 dias na produtividade do meloeiro nos dois ensaios (2009 e 2010). As diferenças observadas nas produtividades entre os dois ensaios podem ser explicadas, para o ano de 2010, pelo fato de ter sido possível somente uma colheita, enquanto foram feitas duas colheitas em 2009. O uso de cobertura do solo aumentou a produtividade do meloeiro, mesmo em período de cultivo considerado inadequado. Analisando a produção do meloeiro nos dois anos de cultivo, observou-se que, em todos os tratamentos, a produtividade foi acima de $38 \mathrm{t}$ / ha, com exceção de SD que obteve 32 t/ha. Para os tratamentos com cobertura do solo houve variação positiva de produtividade, acima da média brasileira, em torno de $104 ; 80 ; 77 ; 71 ; 65$ e $39 \%$ para os tratamentos PDF, BC, PCB, PP, $\mathrm{CC}$ e SD, respectivamente.

No que se refere à qualidade dos frutos (Tabela 2), não houve interação significativa entre tratamentos de cobertura do solo e uso do TNT nos fatores de qualidade estudados, o que também

Tabela 4. Temperatura do solo a 5 e $10 \mathrm{~cm}$ de profundidade, cultivado com meloeiro sob diferentes tipos de cobertura do solo (soil temperature at 5 and $10 \mathrm{~cm}$ deep, cultivated with melon under different types of soil covers). Petrolina, Embrapa Semiárido, $2009 / 2010$.

\begin{tabular}{|c|c|c|c|c|c|c|c|c|c|c|c|c|}
\hline \multirow{3}{*}{$\begin{array}{l}\text { Temperatura } \\
\left({ }^{\circ} \mathrm{C}\right)\end{array}$} & \multicolumn{12}{|c|}{ Ano 2010} \\
\hline & \multicolumn{2}{|c|}{ PDF } & \multicolumn{2}{|c|}{ PP } & \multicolumn{2}{|c|}{$\mathrm{CC}$} & \multicolumn{2}{|c|}{ PCB } & \multicolumn{2}{|c|}{ BC } & \multicolumn{2}{|c|}{ SD } \\
\hline & $5 \mathrm{~cm}$ & $10 \mathrm{~cm}$ & $5 \mathrm{~cm}$ & $10 \mathrm{~cm}$ & $5 \mathrm{~cm}$ & $10 \mathrm{~cm}$ & $5 \mathrm{~cm}$ & $10 \mathrm{~cm}$ & $5 \mathrm{~cm}$ & $10 \mathrm{~cm}$ & $5 \mathrm{~cm}$ & $10 \mathrm{~cm}$ \\
\hline Média & 26,5 & 26,5 & 26,5 & 26,6 & 25,2 & 25,4 & 24,6 & 24,8 & 25,3 & 25,5 & 24,8 & 24,6 \\
\hline Máxima & 28,8 & 28,2 & 28,6 & 28,2 & 26,2 & 26,0 & 25,9 & 25,6 & 26,1 & 26,1 & 26,5 & 27,0 \\
\hline Mínima & 24,5 & 24,9 & 24,5 & 25,0 & 24,3 & 24,7 & 23,7 & 24,0 & 24,5 & 24,9 & 23,2 & 22,5 \\
\hline \multirow[t]{2}{*}{ Amplitude } & 4,3 & 3,3 & 4,1 & 3,2 & 1,9 & 1,3 & 2,2 & 1,6 & 1,6 & 1,2 & 3,3 & 5,5 \\
\hline & \multicolumn{12}{|c|}{ Ano 2009} \\
\hline Média & 27,6 & 27,7 & 26,7 & 26,9 & 26,0 & 26,2 & 25,7 & 26,0 & 26,0 & 26,2 & 25,5 & 25,8 \\
\hline Máxima & 29,8 & 29,3 & 28,8 & 28,3 & 26,8 & 26,8 & 26,8 & 26,6 & 26,8 & 26,7 & 28,5 & 27,6 \\
\hline Mínima & 25,6 & 26,0 & 24,8 & 25,4 & 25,2 & 25,4 & 24,8 & 25,2 & 25,2 & 25,6 & 23,2 & 24,1 \\
\hline Amplitude & 4,2 & 3,3 & 4,0 & 2,9 & 1,6 & 1,4 & 2,0 & 1,4 & 1,6 & 1,1 & 5,3 & 3,5 \\
\hline
\end{tabular}

$\mathrm{PDF}=$ plástico duplo face preto/prata com 30 micra de espessura (black-silvery double face polyethylene film, 30 micra thick); $\mathrm{PP}=$ plástico preto (black polyethylene); $\mathrm{CC}=$ casca de coco seco com $2 \mathrm{~cm}$ de espessura (coconut shell, $2 \mathrm{~cm}$ thick); $\mathrm{PCB}=$ palha de capim buffel (buffelgrass straw); $\mathrm{BC}=$ bagaço de cana (sugarcane bagasse); $\mathrm{SD}=$ solo descoberto sem qualquer tipo de cobertura morta (uncovered soil). 
ocorreu no experimento realizado por Braga et al. (2010). Os tratamentos diferiram nas características de diâmetro longitudinal e espessura da casca, onde o $\mathrm{BC}$ foi superior a $\mathrm{SD}$ e CC, sem diferir dos demais tratamentos. Houve efeito significativo do uso da manta agrotêxtil (TNT) com aumento no diâmetro da cavidade equatorial (DCEq), da cavidade longitudinal (DCLong) e espessura de polpa e casca (Ecasca), porém com redução da espessura da polpa. Como o uso de TNT afetou a ECasca, nos dois ensaios realizados, denota-se que com o uso de TNT pode-se obter uma espessura de casca maior, característica, entre outros, essencial para resistência ao transporte a longas distâncias.

Os valores de SST (média de $10,68^{\circ}$ Brix), $\mathrm{pH}$ (média de 5,56) e acidez total titulável (média de $0,13 \%$ volume de ácido cítrico) dos frutos não mostraram diferenças significativas entre os tratamentos, semelhantemente ao observado por Martins et al. (1998) e Braga et al. (2010). Não houve também qualquer efeito significativo do uso do TNT (dados não apresentados). Os valores médios de SS, pH e acidez para todos os tratamentos estão dentro dos padrões de qualidade da cultivar (Mendlinger \& Pasternak, 1992; Castilhos, 2012), demonstrando a viabilidade de se obter frutos de qualidade, em termos de sabor, em período de cultivo não recomendado, para as condições do Submédio do Vale do São Francisco.

Lâmina de água e índice de produtividade da água (iPA)

A lâmina total de água aplicada via irrigação durante o ciclo de cultivo do melão foi de $269,7 \mathrm{~mm}$ em 2009 e de 239,7 mm em 2010, independente do tratamento. Houve ocorrência de precipitações pluviométricas de 19,1 $\mathrm{mm}$ e $26,2 \mathrm{~mm}$ durante os ensaios nos anos 2009 e 2010, medidos em estação agrometeorologia a cerca de $600 \mathrm{~m}$ da área experimental.

A quantidade total de água usada para produzir um quilograma de melão (iPA) nos diferentes tratamentos, nos cultivos dos anos de 2009 e 2010, são apresentados na Tabela 3. Em 2009, a cobertura do solo com PDF apresentou o menor iPA $(46,42 \mathrm{~L} / \mathrm{kg})$ e o tratamento $\mathrm{SD}(70,41 \mathrm{~L} / \mathrm{kg})$ apresentou o maior
iPA, ou seja, quanto menor o iPA menos água se gastou para produzir um $\mathrm{kg}$ de fruto. Já em 2010, a cobertura do solo com PCB $(62,59 \mathrm{~L} / \mathrm{kg})$ apresentou o menor iPA mantendo o maior valor para o tratamento SD $(93,64 \mathrm{~L} / \mathrm{kg})$. Nos dois períodos de plantio, todos os tratamentos com uso de alguma cobertura do solo obtiveram menores valores, ou seja, foram mais eficientes em utilizar água que o SD. No ano 2009, o tratamento SD utilizou $24 \mathrm{~L}$ de água a mais para produzir um quilograma de melão em comparação para o tratamento PDF; no ano 2010, esse valor foi de $31 \mathrm{~L}$ de água a mais em comparação ao tratamento PCB. Considerando que a maioria das regiões de cultivo comercial do meloeiro no Brasil ocorre no Nordeste, onde há escassez de recursos hídricos, o uso de mulching ou cobertura do solo é prática essencial; além de diminuir custos com capinas (herbicidas), permite obter alta produtividade, produtos de qualidade $\mathrm{e}$ economia de água, devido ao aumento da eficiência do uso da água. Braga et al. (2010), em cultivo nas mesmas condições edafoclimáticas, porém em período de cultivo tradicional (setembro), obtiveram menores valores de iPA nos cultivos realizados no mês de junho de 2009 e 2010. Embora a quantidade de água usada tenha sido maior no período de cultivo tradicional, também, foi maior a produtividade, fato que pode explicar os diferentes resultados encontrados.

\section{Variação de temperatura do solo}

Umas das grandes preocupações com o uso de coberturas do solo é a temperatura, ou seja, a dinâmica da variação da temperatura que pode comprometer o desenvolvimento das culturas, principalmente em regiões com alta incidência solar, caso do Nordeste brasileiro. Os valores das temperaturas máximas e mínimas, nas profundidades de 5 e $10 \mathrm{~cm}$, foram maiores nas condições de cobertura do solo com material plástico (PDF, PP) comparados aos materiais orgânicos (Tabela 4). Foram verificadas as maiores variações de amplitude térmica dos tratamentos com cobertura plástica do solo em relação aos materiais orgânicos, PCB, BC e CC. Esse resultado pode ser um fator determinante para a escolha do tipo de mulching a ser usado no cultivo do meloeiro. Em regiões com temperaturas altas, como as dos presentes ensaios, os tratamentos de cobertura (plásticos) usados demandaram maiores replantios de mudas, apesar das temperaturas do solo entre 5 e $10 \mathrm{~cm}$ de profundidade serem semelhantes; esse fato pode ser explicado pela proximidade entre os pontos de tomada das medidas. Houve maiores variações na amplitude das temperaturas nos tratamentos SD, PDF e PP. De acordo com os dados analisados, ressalta-se também, que o perfil de temperatura do solo, a 5 e $10 \mathrm{~cm}$ de profundidade, não foi afetado pelo uso ou não do TNT.

Os resultados mostram que é possível o cultivo do meloeiro tipo amarelo no período e nas condições edafoclimáticas dos ensaios, obtendo rendimentos e qualidade de frutos aceitáveis, tanto para o consumo interno como para exportação. Considerando as produtividades nos dois ciclos de cultivo, os tratamentos que sobressaíram em ordem decrescente foram PDF; BC; PCB; PP, CC e SD. Em termos de índice de produtividade da água (iPA), considerando os valores totais nos dois ciclos de cultivo, os tratamentos que usaram menos água por $\mathrm{kg}$ de fruto, em ordem decrescente foram PDF; BC; PCB; PP; CC e SD.

\section{AGRADECIMENTO}

Aos assistentes e técnicos da Embrapa Semiárido pelo auxílio na condução desse experimento no campo experimental de Bebedouro, em Petrolina-PE. À FACEPE (Fundação de amparo à ciência e tecnologia do estado de Pernambuco) pela concessão de bolsas.

\section{REFERÊNCIAS}

AGRIANUAL. 2013. Anuário da agricultura brasileira. São Paulo: FNP Consultoria e Agro Informativos, $480 \mathrm{p}$.

AMARIZ, A; LIMA, MAC; BRAGA, MB; TRINDADE, DCG; RIBEIRO, TP; SANTOS, ACN. 2009. Conservação pós-colheita de melão cultivado com diferentes tipos de cobertura do solo e com uso de manta agrotêxtil. In: CONGRESSO BRASILEIRO DE OLERICULTURA, 49, Águas de Lindóia. Anais....Brasília: Horticultura Brasileira 27: S548-S544. 
BATTIKHI, AM; GHAWI, I. 1987. Muskmelon production under mulch and trickle irrigation in the Jordan Valley. Hortscience 22: 578-581.

BRADENBERG, L; WIENDEFELD, B. 1997. Physical characteristics of mulches and their impact on crop response and profitability in muskmelon production. HortTechnology 7: 165-169.

BRAGA, MB; RESENDE, GM; MOURA, MSB; DIAS, RCS; COSTA, ND; CALGARO, M; CORREIA, JS; SILVA, FZ. 2010. Produtividade e qualidade do melão submetido a diferentes tipos de cobertura do solo. IRRIGA 15: 422-430

CÂMARA, MJT; NEGREIROS, MZ; MEDEIROS, JF; BEZERRA NETO, F; BARROS JÚNIOR, AP. 2007. Produtividade e qualidade de melão amarelo influenciado por coberturas do solo e lâminas de irrigação no período chuvoso. Ciência Rural 37: 58-63.

CASTILHOS, LF. 2012. Cultivo de melão e melancia. Curitiba: Tecpar, $33 \mathrm{p}$.

CRISÓSTOMO, AC; SANTOS, AA; RAIJ, B; FARIA, CMB; FERNANDES, FAM; SANTOS, FJS; CRISÓSTOMO, JR; FREITAS, JAD; HOLANDA, JS; CARDOSO, JW; COSTA, ND. 2002. Adubação, irrigação, híbridos e práticas culturais para o meloeiro no Nordeste. Fortaleza: Embrapa Agroindústria Tropical, 21p. (Circular técnica, 14).

DIAS, RCS; SILVA, CMJ; COSTA, ND; FARIA, CMB; LIMA, MAC; SANTOS, MH; SOARES, JM; HAJI, FP; ASSIS, JS; PAIVA, LB; BARBOSA, GS; MEDEIROS, KN. 2006. Desempenho de melão tipo amarelo em diferentes coberturas de solo e sob cultivo temporariamente protegido no Vale do São
Francisco. In: CONGRESSO BRASILEIRO DE OLERICULTURA, 46. Anais...Goiânia: SOB. Horticultura Brasileira 24. Suplemento CD-ROM.

FERREIRA, DF. 2008. SISVAR: um programa para análises e ensino de estatística. Revista Cientifica Symposium 6: 36-41.

INSTITUTO ADOLFO LUTZ. 1985. Normas analíticas, métodos químicos e fisicos para análise de alimentos. 3.ed. São Paulo: Instituto Adolfo Lutz, v. 1, 533p.

JENSEN, ME. 2007. Sustainable and productive irrigated agriculture. In: HOFFMAN, GJ; EVANS, RG; JENSEN, ME, MARTIN, DL, ELLIOTT, RL (eds). Design and operation of farm irrigation systems. 2.ed., St. Joseph: ASABE. p. 33-56.

MARTINS, SR; PEIL, RM; SCHWENGBER, JE; ASSIS, FN; MENDEZ, MEG. 1998. Produção de melão em função de diferentes sistemas de condução de plantas em ambiente protegido. Horticultura Brasileira 16: 24-30.

MEDEIROS, JF; SILVA, MCC; CÂMARA NETO, FG; ALMEIDA, AHB; SOUZA, JO; NEGREIROS, MZ; SOARES, SPF. 2006. Crescimento e produção de melão cultivado sob cobertura do solo e diferentes frequências de irrigação. Revista Brasileira de Engenharia Agrícola e Ambiental 10: 792-797.

MENDLINGER, S; PASTERNAK, D. 1992. Effect of time of salinization on flowering, yield and fruit quality factors in melon, Cucumis melo L. Journal of Horticultural Science 67: 529-534.

MIRANDA, NO; MEDEIROS, JF; NASCIMENTO, IB; ALVES, LP. 2003. Produtividade e qualidade de frutos de melão em resposta à cobertura do solo com plástico preto e ao preparo do solo. Horticultura Brasileira 21: 490-493.

MOURA, SB; SOUZA, LSB; BRAGA, MB; BRANDÃO, EO; CORREIA, JS, CARMO, JFA; SILVA, FZ; SILVA, TGF. 2009. Temperatura do solo cultivado com meloeiro sobre diferentes coberturas do solo no Submédio São Francisco. In: CONGRESSO BRASILEIRO DE AGROMETEOROLOGIA, 16. Anais... Belo Horizonte: SBA: UFV: Embrapa Milho e Sorgo.

OROZCO, SM; PREZE, ZO; LOPEZ, AO. 1995. Effect of transparent mulch on insect populations, virus diseases, soil temperature, and yield of cantaloup in the tropical region. New Zealand Journal Crop Horticultural Science 23: 199-204.

PIRES, MML; SANTOS, HA; SANTOS, DF; VASCONCELOS, AS; ARAGÃO, CA. 2013. Produção do meloeiro submetido a diferentes manejos de água com o uso de manta de tecido não tecido. Horticultura Brasileira 31: 304-310.

SANTOS, FGB. 2012. Análise do crescimento, produção e qualidade de melão cantaloupe, em cultivo protegido temporariamente com agrotêxtil em Mossoró-RN. Mossoró: UFERSA. 137p. (Tese doutorado).

SOUSA, VF; COELHO, EF; ANDRADE JUNIOR, AS.; FOLEGATTI, MV.; FRIZZONE, JA. 2000. Eficiência do uso da água pelo meloeiro sob diferentes frequências de irrigação. Revista Brasileira de Engenharia Agrícola e Ambiental 4: 183-188. 